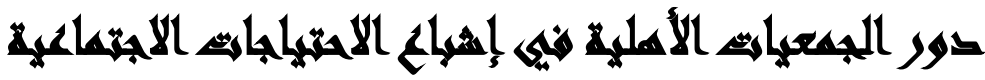

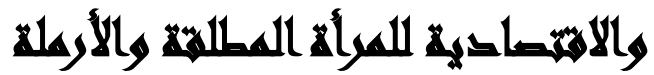

[?]

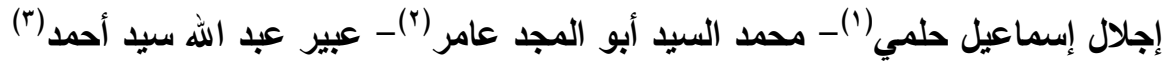

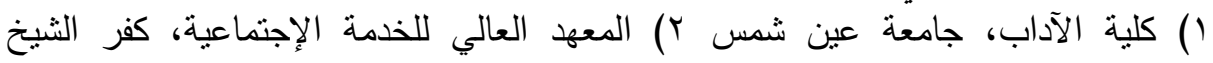

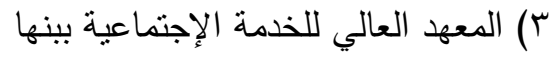

\section{المستخلص}

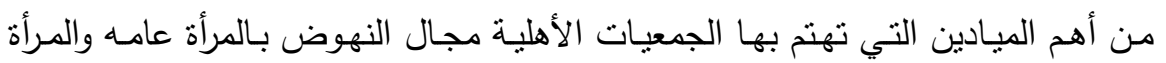

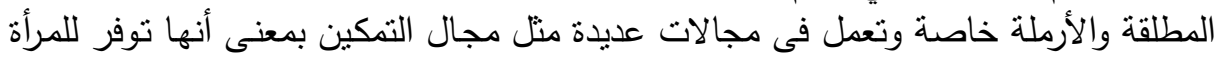

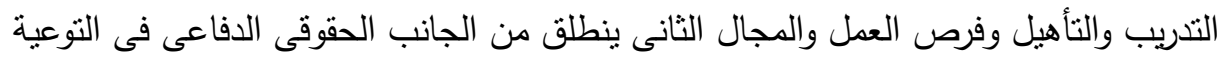

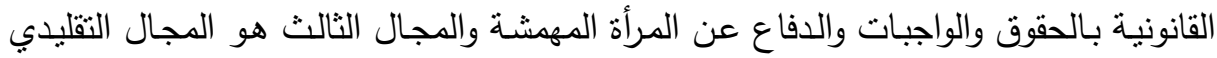

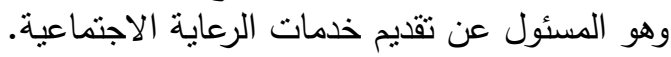

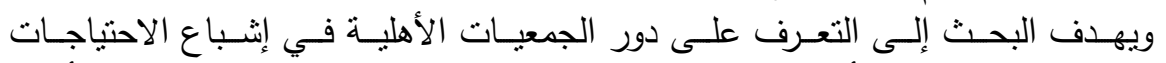

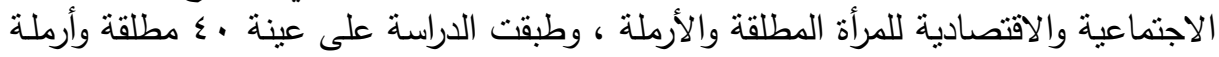

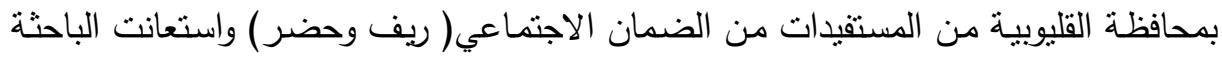

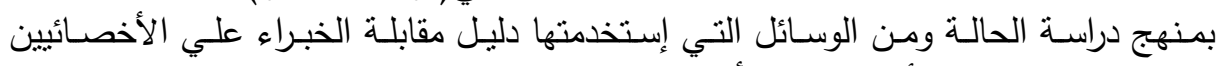

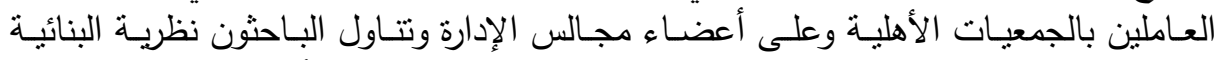

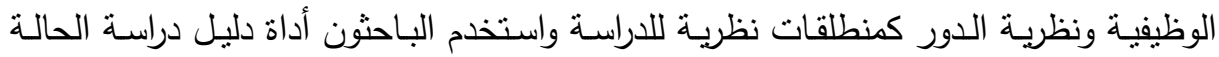
على المطلقات والأرامل. قد توصلت الدراسة إلى نتائج هامة منها:

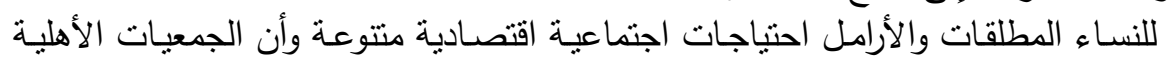

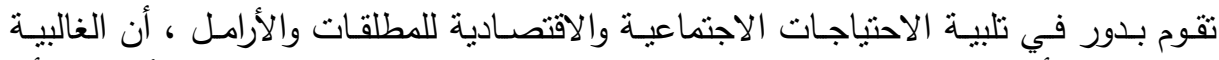

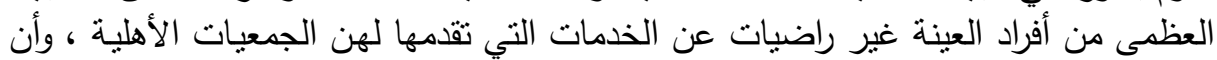

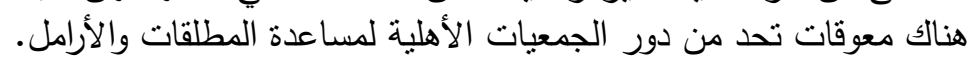

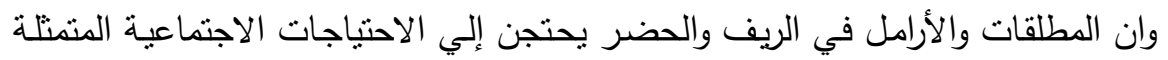

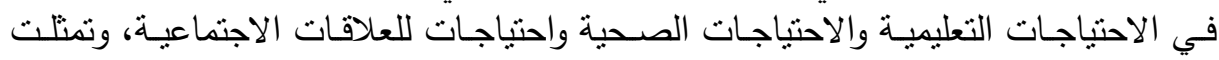

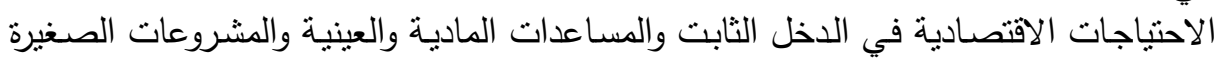

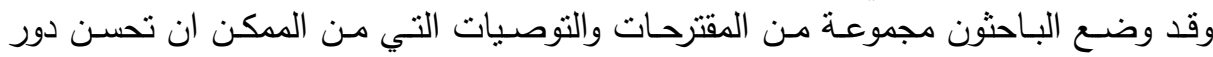

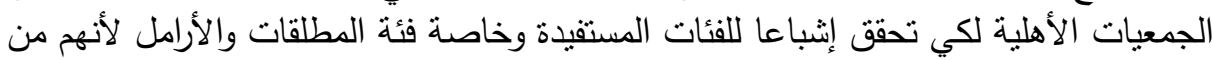

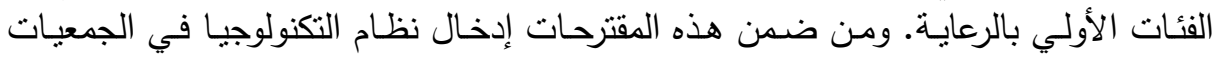

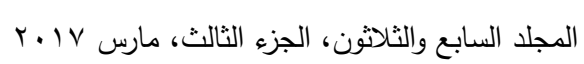


الأهلية وتحسين آليات الجمعيات الأهلية من خلال عمل دورات تدريبية للعاملين بها وتحسين الجان الجين مستوي الخدمة المقدمة والأنشطة التي تقوم بها الجمعيات الأهلية وذللك من خلال عمل الأل أبحاث علمية بصفة مستمرة لمعرفة الاحتياجات الفعلية للفئات المستقيدة.

\section{ranodl}

يشهر المجتمع الهصري في الوقت الراهن تغيرات شديدة الخطورة أطاحت معها بكل القيم والواجبات، وضاعت معها كل الاهتمامات وكان لابد من تكاتف جميع مؤسسات الدولة لتلبية

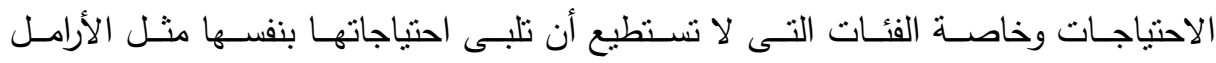

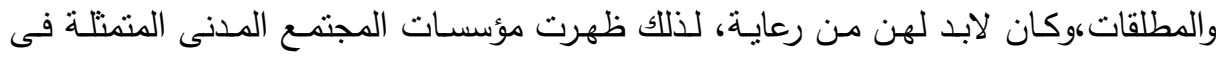

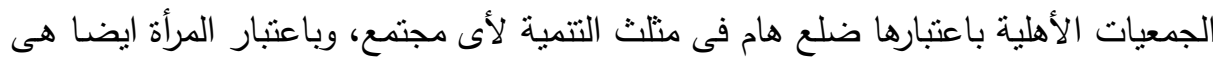
نصف المجتمع فإذا ارتقينا بها سوف نرتقى بالمجتمع بأسره.

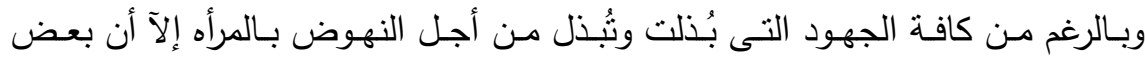

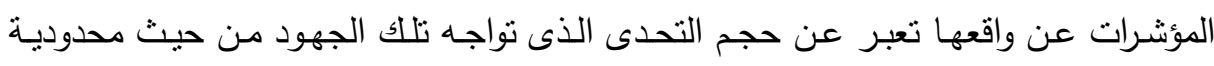

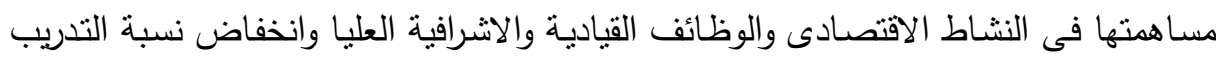

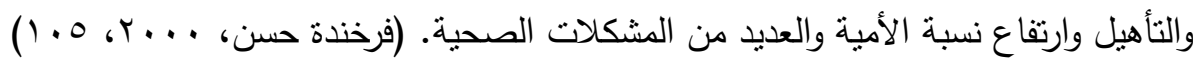

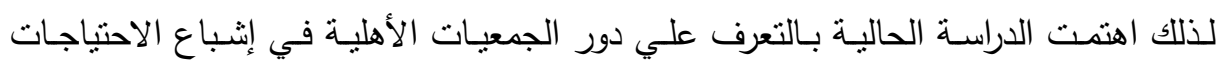
الاجتماعية والاقتصادية للمطلقات والأرامل.

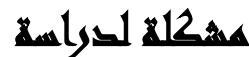

تواجه المرأة العديد من الأوضـاع المجتمعية السيئة، التى تنعكس عليها وتجعلها تتولى

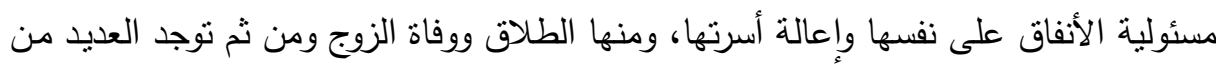

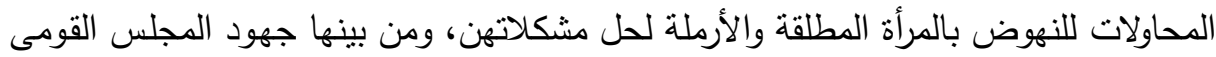

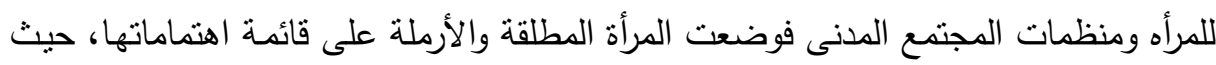

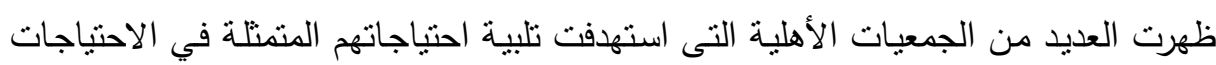

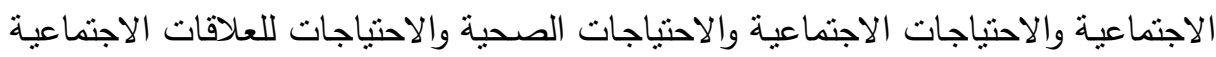

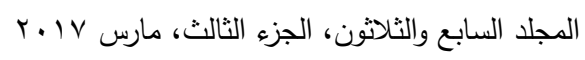


والاحتياجات الاقتصادية المتمنلة في الحاجة الي الدخل الثابت والحاجة للمساعدات والمعونات

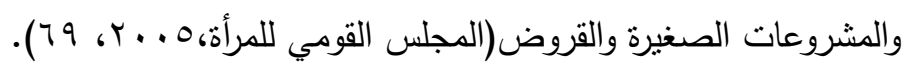

أن مؤسسات المجتمع المدنى المتمثله فى الجمعيات الأهلية تؤدي دورا أساسيا فى التقليل

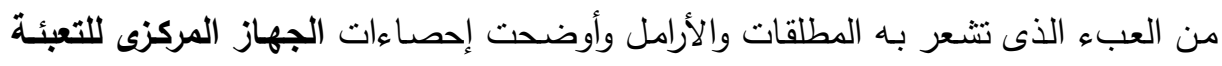

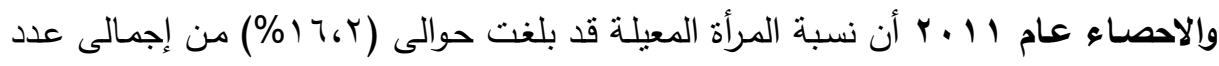

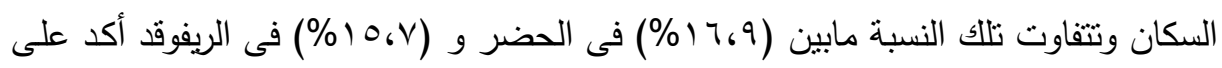

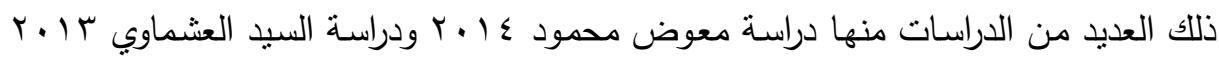

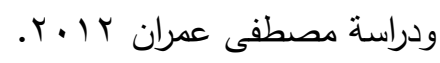
وتتبلور مشكلة الدراسة الحالية في تساؤل رئيسي مؤداه، ما دور الجمعيات الأهلية في إثباع

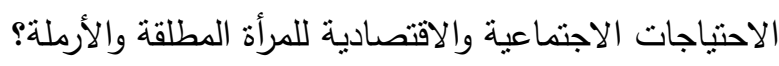

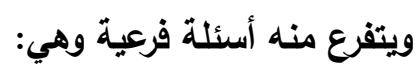

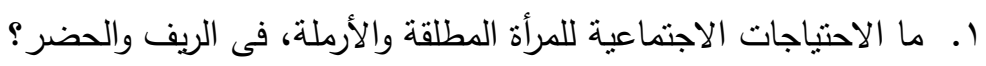

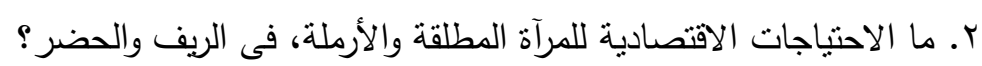

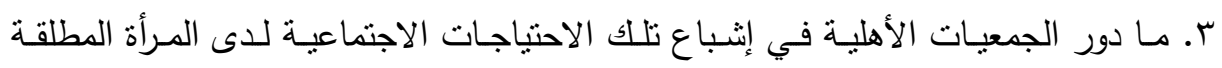

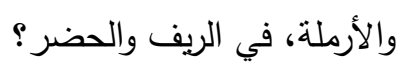
ع. ما دور الجمعيات الأهلية في إثباع الاحتياجات الاقتصادية لدى المرأة المطلقة والأرملة،

$$
\text { في الريف والحضر؟ }
$$

ه. ما المعوقات النى تحد من دور الجمعيات الاهلية في إشباع الاحتياجات الاقتصادية لاى المرأة المطلقة والأرملة، في الريف والحضر؟ دُ دورج

\section{أهمية السوراسما}

من الناحية النظرية تتمثل في: - - دراسة الاحتياجات الاجتماعية والاقتصادية التي تواجه المرأة المطلقة والأرملة، حيث نجد فئ قلة في الدراسات التي اهتمت بدراسة هذه الاحتباجات وكيفية إثباعها. 
- التعرف على دور الجمعيات الأهلية في تلبية الاحتياجات الاجتماعية والاقتصـادية التي تواجه المرأة المطلقة والأرملة.

- العمل على توفير وتلبية الاحتياجات الاجتماعية والاقتصادية للمرأة وخاصسة فئة الأرامل والمطلقات فالمرأه تمنل نصف الطاقة البشرية. ومن الناحية التطبيقية وتتمثل في: - في ضوء مـا يتوصل إليه الباحثون من نتائج قد تكون لهذه الدراسـة مساهمة فى تغيير التشريعات التى تعوف تلبية الاحتياجات الاجتماعية والاقتصادية الخاصة بالمرأة. - قد تكون هذه الدراسة نواة لبحوث أخرى مستقبلية في هذا المجال.

\section{أهساهنغ التوراسما}

للارسـة هدف رئيسي يتمثل في: " التعرف على دور الجمعيات الأهليـة في إثشباع

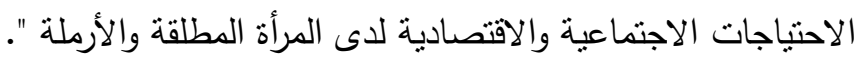
مفاهيم الاراسة: 1 - مفهوم الجمعيات الأهلية: يتحدد مفهوم الجمعيات الأهلية فى الآتى: - بأنها كيان منظم يهدف لتحقيق أغراض معينة ويتمتع بشخصية معنوية.

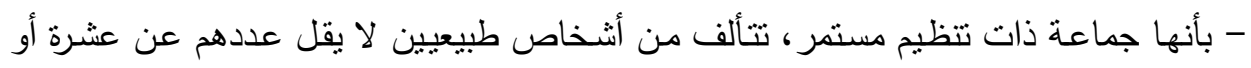
من أنثخاص اعتباريين. - أنها منظمات أنشئت لتحقيق أهداف اجنماعية معينة. - بأنها وحدات اجتماعية ينم بناؤها بشكل مقصود لتحقيق أهداف محددة يعجز الجهد الفردي اهي

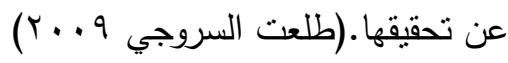
r - مفهوم كلمة إثباع تشير كلمة إثباع إلى مدى الرضا لدى الفئات المستقيدة من خدمات

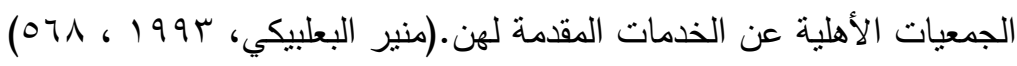




$$
\text { r- مفهوم الاحتياجات الاجتماعية والاقتصادية: }
$$

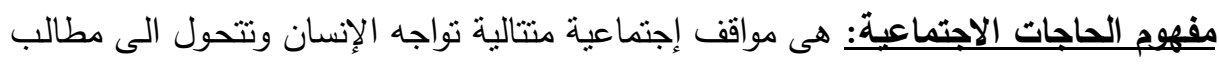

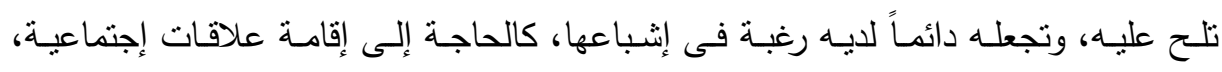
والحاجة إلى التعليم والصحة والمسكن والمعاملة الطيبة من المحيطين.

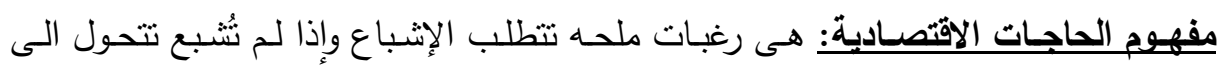

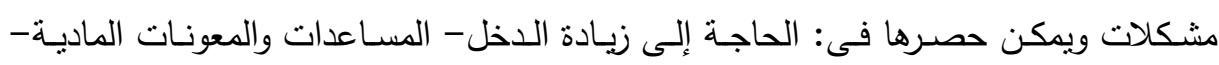

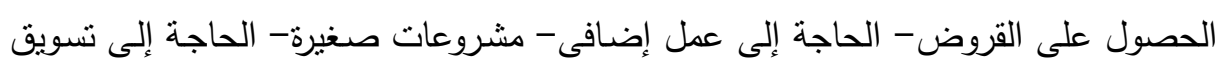

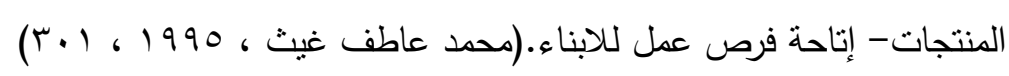

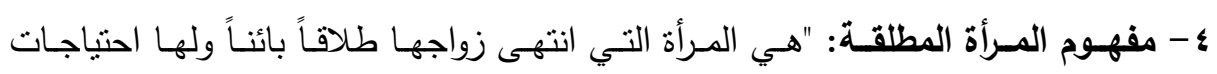

$$
\text { اجتماعية واقتصادية. }
$$

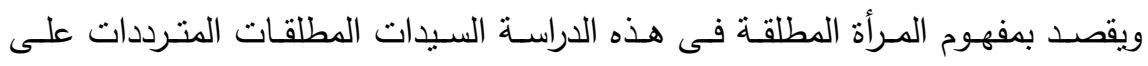

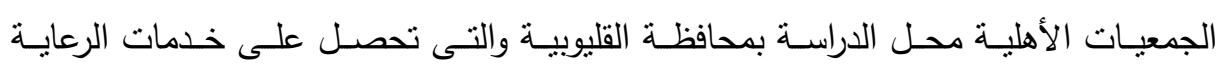
الاجتماعية والاقتصادية منها. ه- مفهوم المرأة الأرملة: هي المرأة التي فقدات زوجها وليس لها أي عائل أو مصدر للاخل

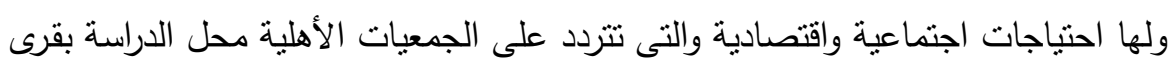

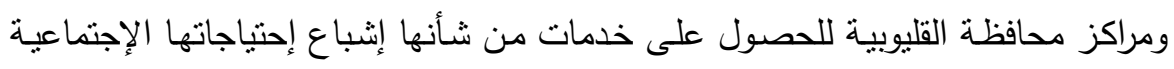

$$
\text { والإقتصادية (علي الطهطاوي، .... ب ، } 7 \text { ( ). }
$$

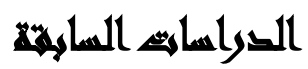

- دراسة (أحمد محمد حسن محمد، 10 ـ ب) بعنوان : دور الجمعيات الأهلية فى تحقيق الحماية الاجتماعية للققراء بالريف بمحافظة قنا وهدفت الدراسة إلى تحديد دور

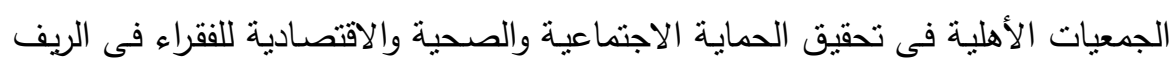

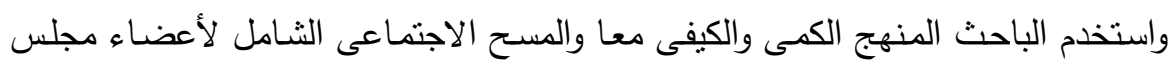

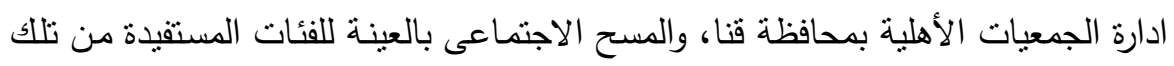

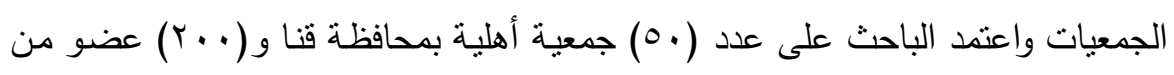

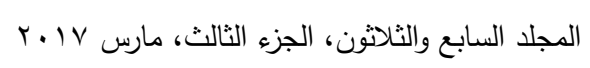


أعضـاء مجلس الادارة و( . ؛ ) عضو من المستقيدين من الجمعيات الأهلية وتوصلت

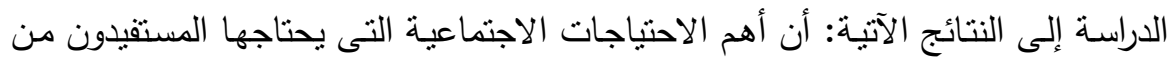

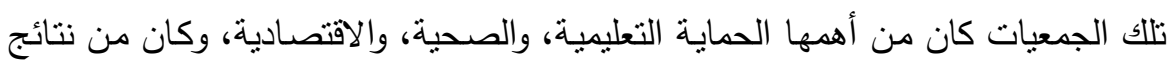

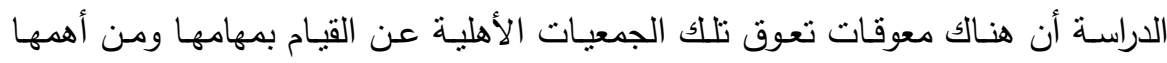
ضعف التمويل والروتين ونقص الخدمات، و من أهم الفئات المستفيدة بتلك الجمعيات هي الهى فئة الأرامل والمطلقات.

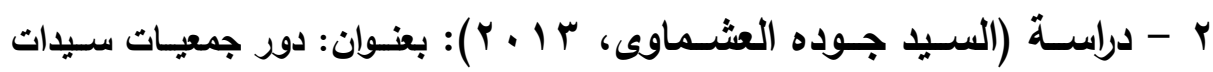
الأعمـال كإحدى الجمعيات الأهليـة فى مواجهة فقر المرأة دراسـة مطبقة على جمعيـة سيدات الأعمال بالقاهرة"، وهدفت الدراسة إلى التعرف على الدور الذئ تقوم بـه جمعيات سيدات الأعمال فى مواجهة فقر المرأة وتحديد المعوقات التى تواجه المرأة الفقيرة وكيفية الاستفاده من الخدمات والبرامج التى تقدمها جمعيات سيدات الأعمال فى مواجهة الفقر ولهر واتبعت الدراسـة مــنهج دراسـة الحالـة والمســح الاجتمـاعى بنوعيـة (الثـامل، وبالعينـة)

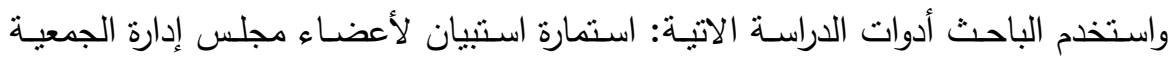
المصرية لسيدات الأعمال بالقاهرة والذى بلـغ عددهن (0) سيدات من سيدات النّات الأعمال

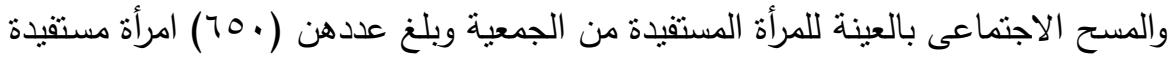

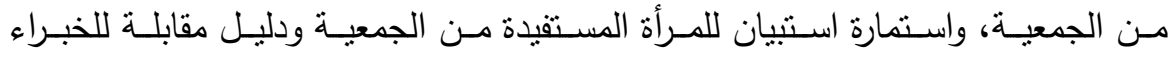

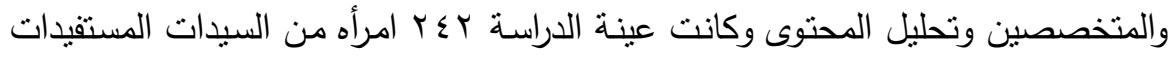

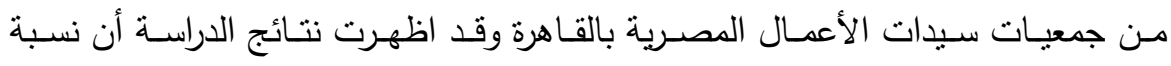

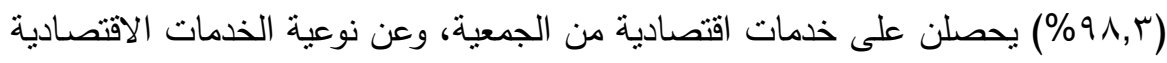
التى حصلت عليها السيدات المستقيدات من الجمعية على النحو التالى: تقدم الجمعيـة

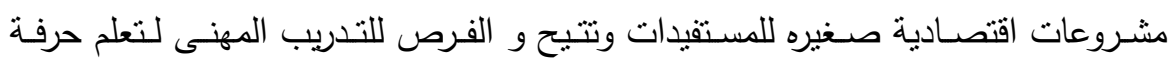
تتاسب احتياجاتهن فى سوق العمل وتدريبهن على المهارات المطلوبـة لتحسين قدراتهن

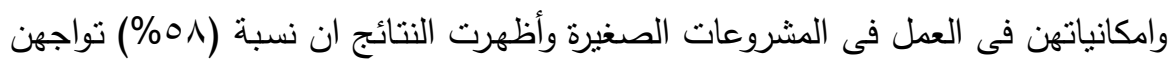
بشكل اساسى معوقات. 


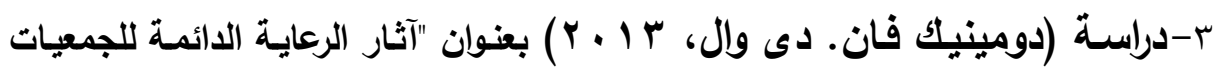

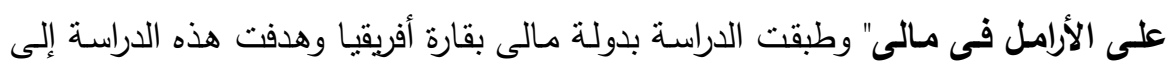

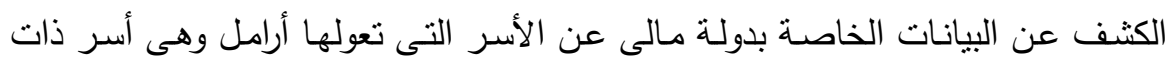

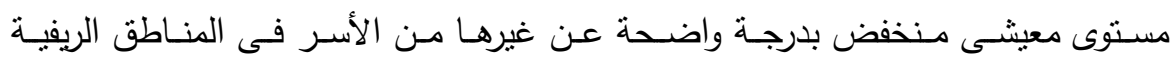

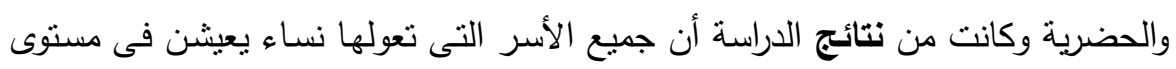
معيثى متدنى من الناحية الاقتصادية عن بقية الأسر الأخرى.

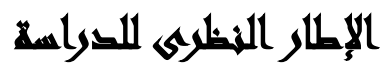

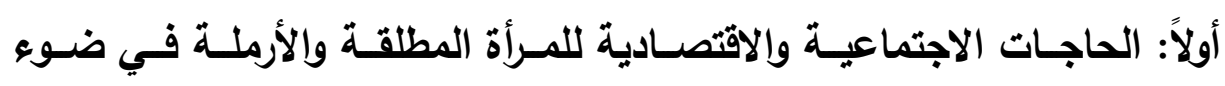

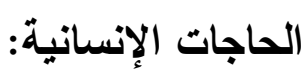

1- الحاجات الاجتماعية: وهي عبارة عن الحاجة إلى تحمل المسئولية الاجتماعية من جانب الإسيا

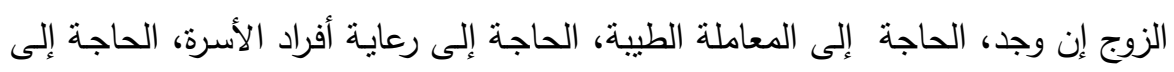

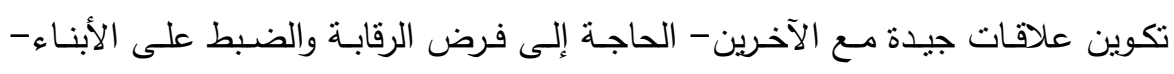

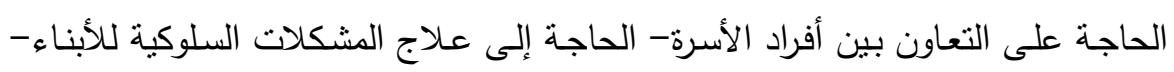

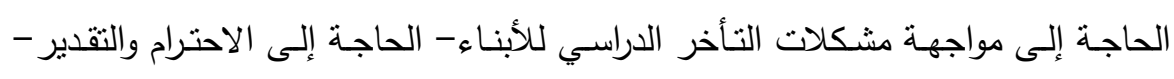

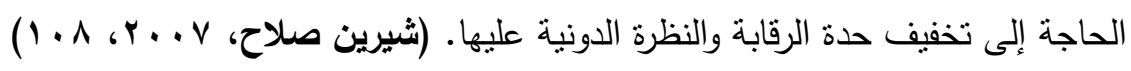

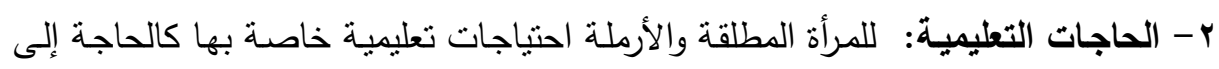
برامج لمحو أميتها وبرامج التأهيل والتدريب الحرفى والحاجة إلى تتمية المهارات لمزاولة

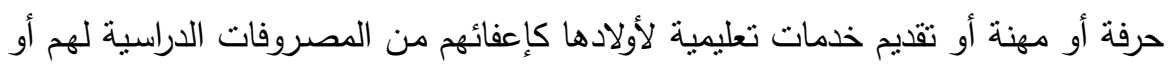

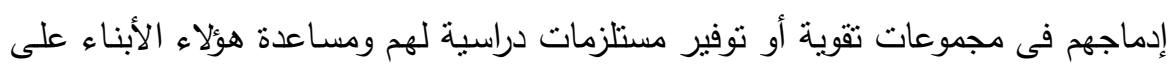
الاستفادة من خدمات الرعاية الإجتماعية التى تقدمها المدرسة. r- الحاجات الصحية: الرعاية صحية لهن مثل عمل تأمين صحى وتوفير مستشفيات على الإنى مستوى عالى من الرعاية وكذلك توفير العلاج بالمجان لهن والكثف الدورى كل فترة زمنية بأجر رمزى أما عن الاحتياجات الصحية لأبناء المطلقات والأرامل فهى تتحصر فئل فئ تقديم

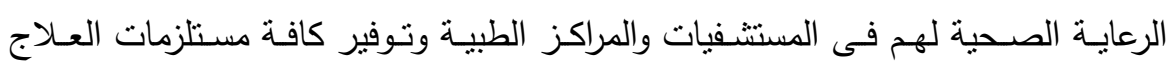

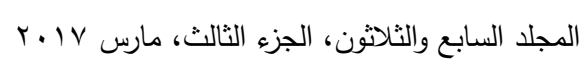


بالمجان والاهتمام بالتطعيمات اللازمة لصغار السن منهم وتيسير اجراء العمليات الجراحية

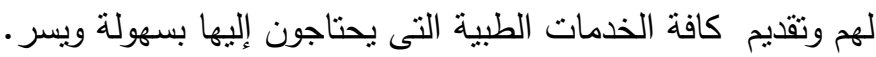

ع - الحاجـة للمسكن: يعد المسكن مـن أهـ الاحتباجـات الاجتماعيـة التى تحتاجهـا المـرأة

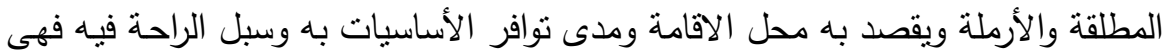

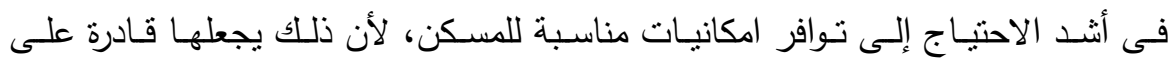

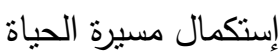
ه - الحاجات الاقتصادية: أما عن الاحتباجات الاقتصادية للمرأة المطلقة والأرملة تتمثل في:

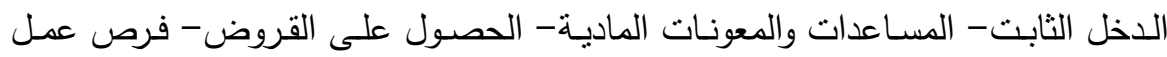
مناسبة وجيدة- مشروعات صغيرة- الحاجـة إلى تسويق المنتجات- اتاحـة فرص عمل للأبناء.

$$
\text { ثانيا: الجمعيات الأهلية (تعريف - أهمية - أهداف): }
$$

الجمعيات الاهلية هى تلك المنظمات التى تقوم على الجهود التطوعية لجماعات من الافراد المهنمين بالخدمـه العامـة يقومون بتتظيمها وإدارتها فى إطـار النظـام العـام والقوانين

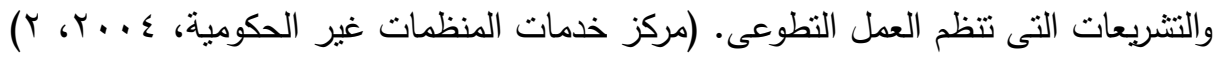
أهمية الجمعيات الأهلية: أنها تساهم بطريقة غير مباثرة فى تلبية الاحتباجات الاقتصادية

للفئات المحتاجة. (Britta Sadoun, 2005) أهداف الجمعيات الأهلية: تهدف الجمعيات الاهلية الى تحقيق أهداف عامة تتمثل فى تحقيق رسـالتها التتمويـة فـى المجتمع فهى تسعى الـى تحقيق انتمـاء تقـافى واجتمـاعى واقتصــادى

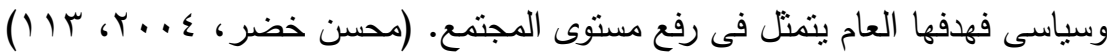

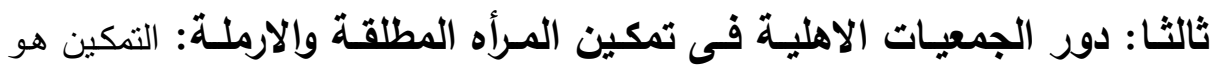
عملية مساعدة الأفراد ليكتسبوا القدرة على اتخاذ القرارات والتصرف فى حياتهم أو مشكلاتهز كما يريدون من خـلال التأثير على جملة المواقف الاجتماعية والثخصية التى يمرون بها.

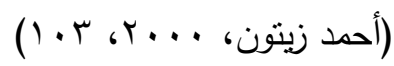


وتمكين المرأة هو تفعيل دورها من خـلال تحسين أحوالها وأحوال أسرتها المعيشية فى النواحى الاجتماعية والاقتصـادية والصحية والتعليمية والمستوى التقافى والسياسى، والتهين والتمكين

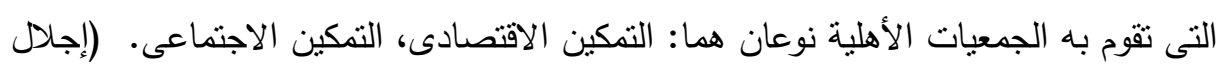

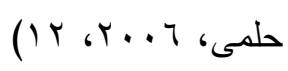
رابعـا: المعوقات التـى تعوق الجمعيـات الاهليـة عن إثـباع احتياجـات المـرأة المطلقة والارملة. - عدم التتسيق بين الجمعيات الأهلية فى نقديم الخدمات. - قلة البحوث التى تجريها الجمعيات الأهلية فى البحث عن الاحتياجات الحقيقية للمستقيدين بما يناسب كل زمان ومكان. - عدم تطوير آليات تقديم الخدمات فى تلك ان الجمعيات. - إهمال الجانب التكنولوجى فى الجمعيات الأهلية. - - إهمال الجانب الاجتماعى والدينى للفئات المستقيدة.

\section{إلجراعاهي القواسة}

تتنمي هذه الدراسة إلي الدراسات الوصفية التحليلية المقارنة كما أنها لا نقف عند مجرد

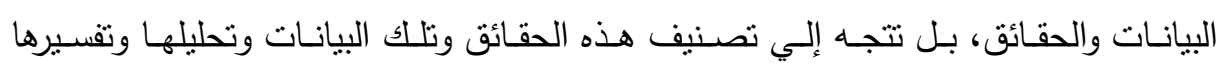
لاستخلاص دلالاتها، وتحديدها بالصورة التي عليها كما وكيفا بهدف الاجابة علي نساؤلات

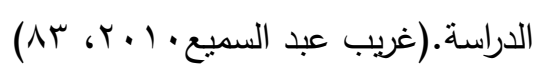

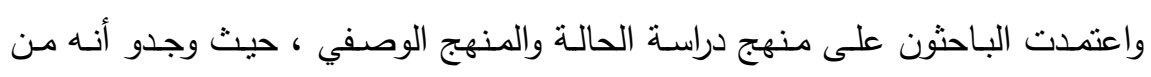

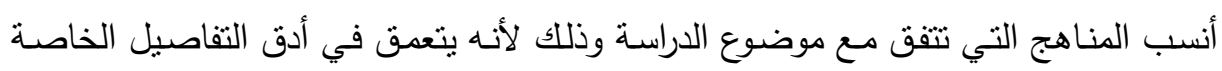
بالدراسة، والعينة. 


\section{مجالايت السراسمة}

أ- المجـال المكـاني للاراسـة: لقد اختارت الباحثة محافظة القليوبيـة(مركز بنها وشبرا شـرق

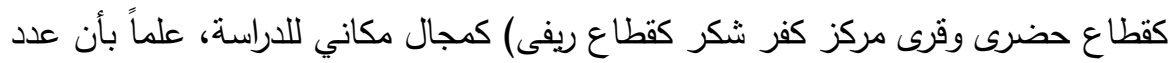

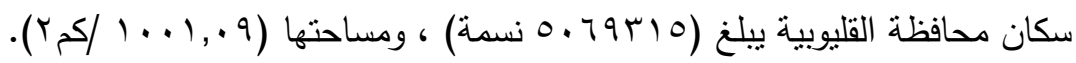

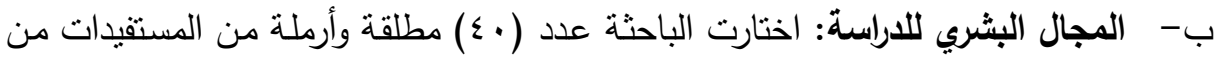
الجمعيات الاهلية النى نم إختيارها للتطبيق بها بمحافظة القليوبية.

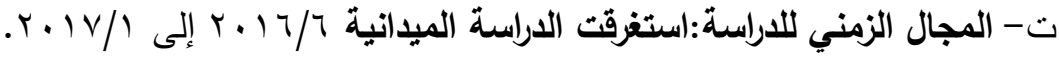

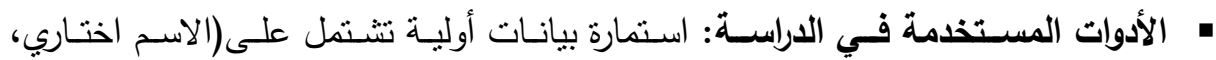

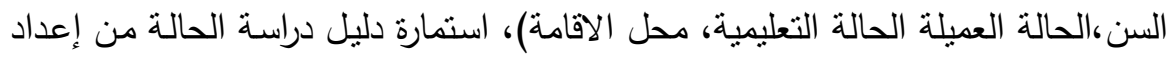
الباحثون على المطلقات والأرامل المستفيدات من الجمعيات الاهلية محل الدراسة.

\section{نمأئج الصراسلة}

توضـح نتائج الدراسـه أن الغالبيـه العظمى من عينه الدراسـه ثقع فـى الفئه العمريـه

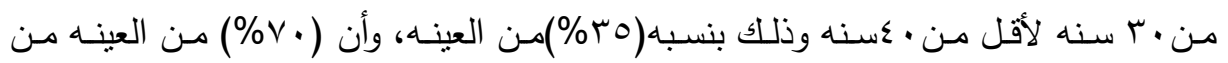

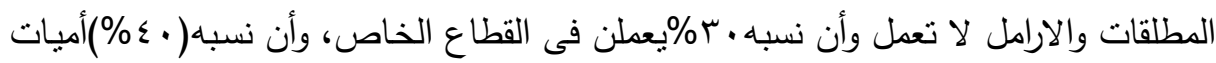

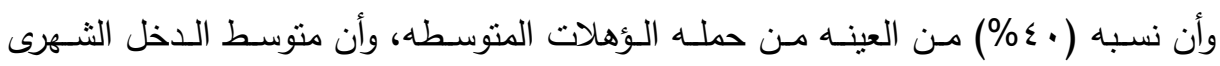

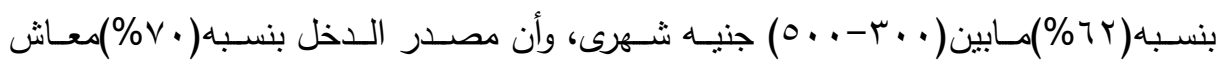

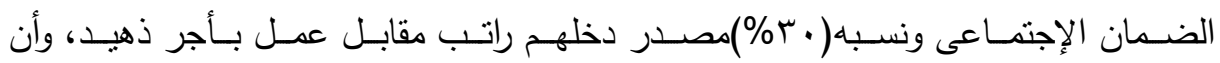

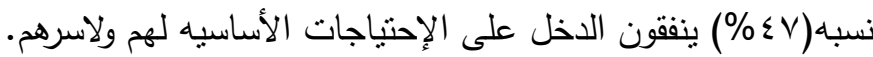
نتائج الدراسة وفقا لتساؤلات الدراسة: التساؤل الأول: ما هى الاحتياجات الاجتماعية التى تواجة المرآه المطلقة والارملة، فى الريف

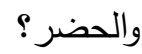
أثنارت نتائج الدراسة الحالية إلي أنه هناك العديد من الاحتباجات الاجتماعيه التي

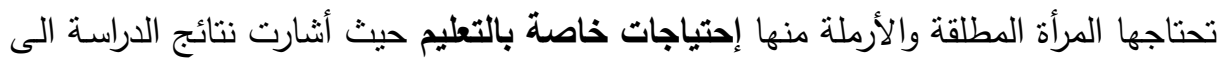

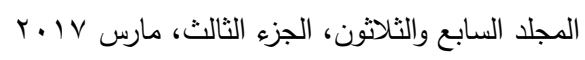




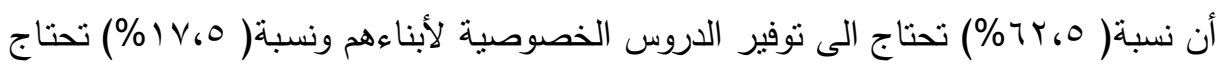

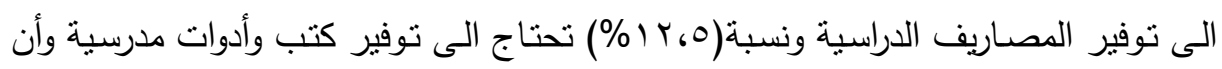

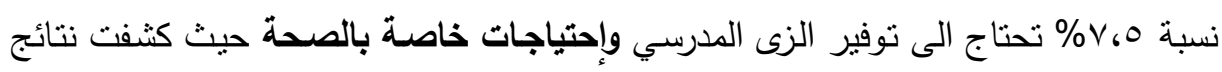

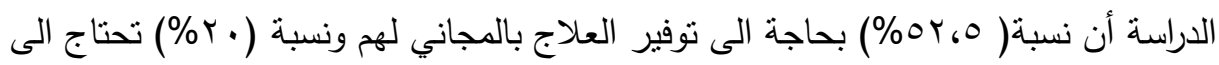

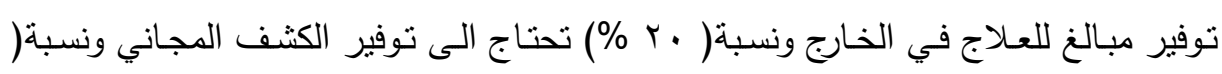

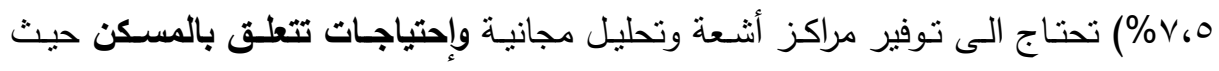

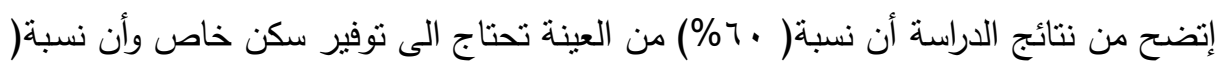

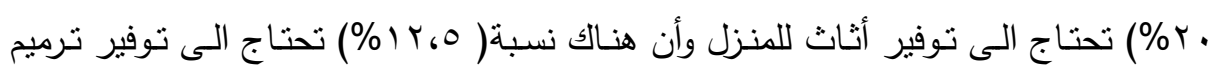

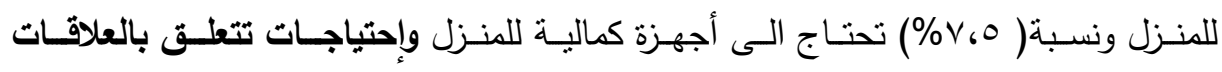
الإجتماعيـة حيث جاء فى الترتيب الاول بنسبة (

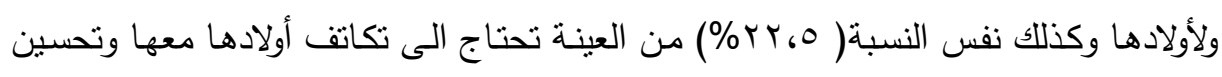

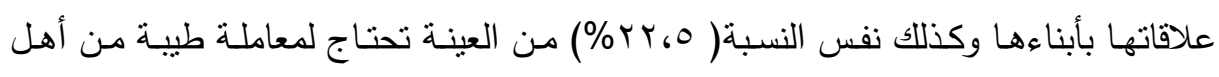

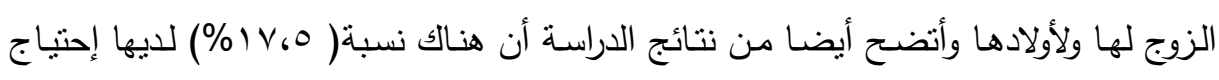

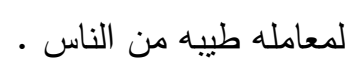
التساؤل الثاني: ما هى الإحتياجات الإقتصادية التى نواجة المرآه المطلقة والارملة، فى الريف والحضر؟

اتضح من الدراسة أن نسبة( . . 1\%) من العينة من المطلقات والأرامل تحتاج الى دخل ثابت في الريف والحضر ومساعدات ومعونات من الجمعيات الأهلية في الريف والحضر ،

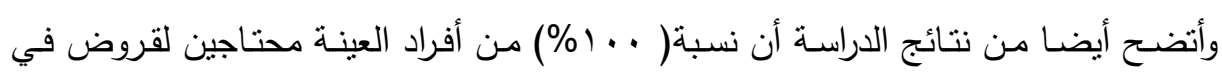

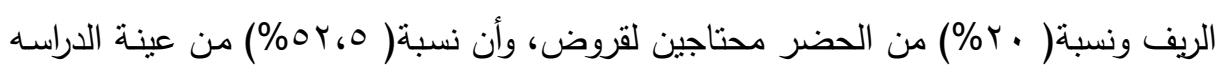
من المطلقات والأرامل تحتاج الى المشروعات الصغيره في الريف والحضر .

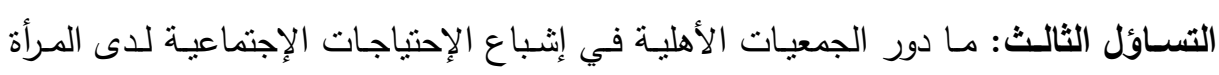

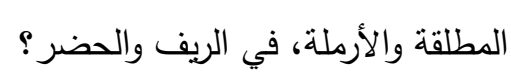

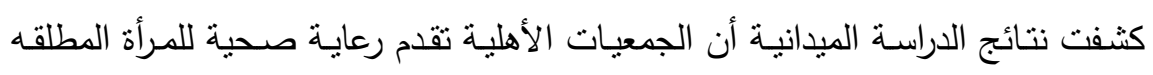

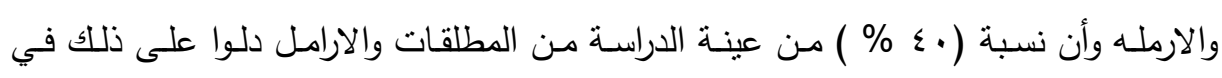


الريف والحضر ، بينما جاءت في الترتيب الثاني بنسبة (r.0 \% \% ) إلى أن الجمعيات الأهلية

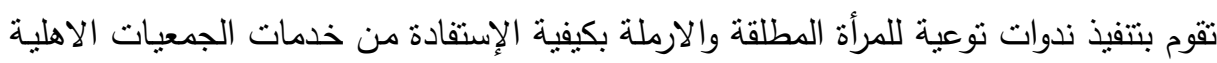

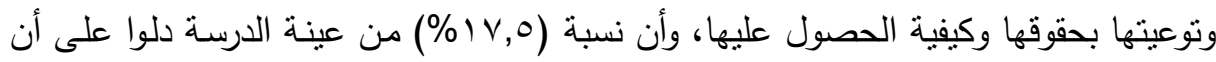
الجمعيات الأهلية تقوم بتتفيذ دورات تدريبية للمرأة المطلقة والارملة.

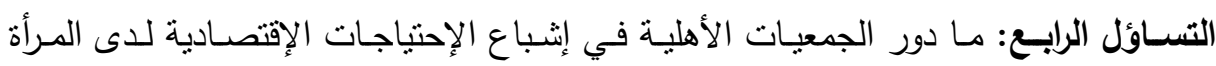

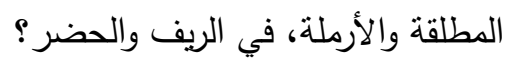
تبين من خـلا الدراسـة الميدانيـة من خلال المقابلات مـع المطلقات والأرامل عينة

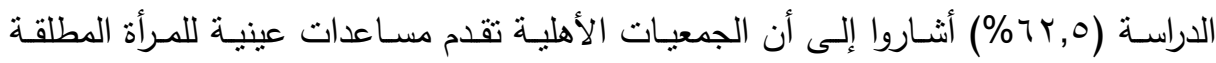

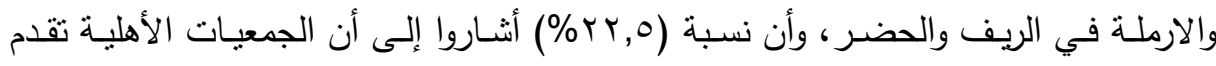

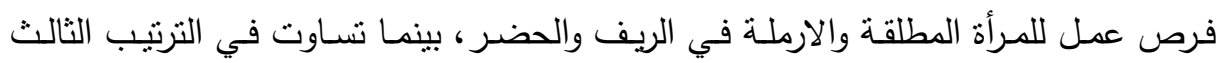

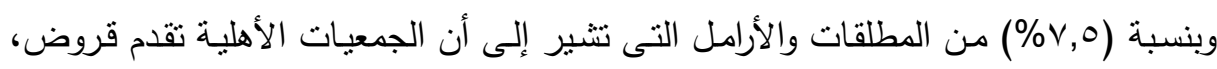

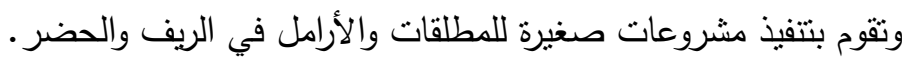

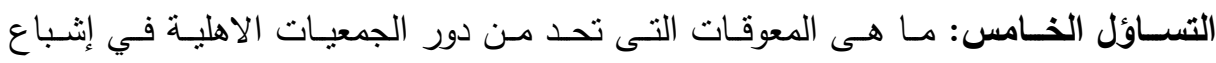

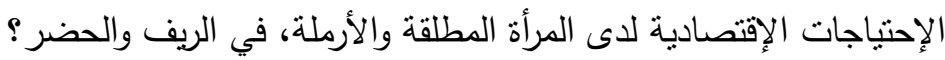

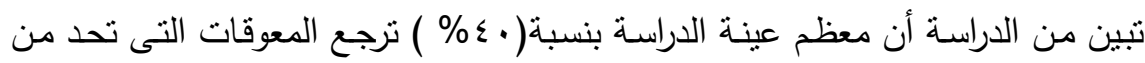

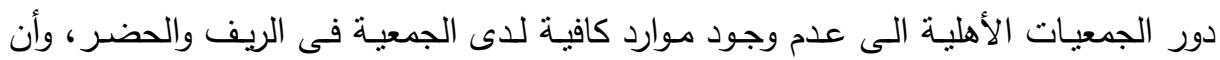

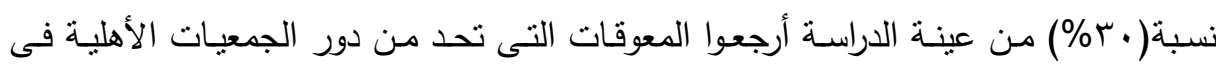
إثباع إحتياجاتهم إلى عدم وجود تقدير فعلى للإحتياجات التى يحتاجون اليها فى الريف

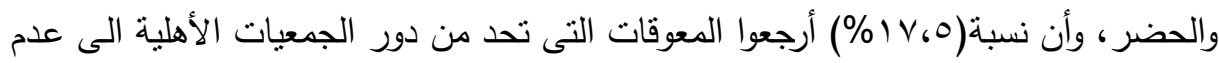

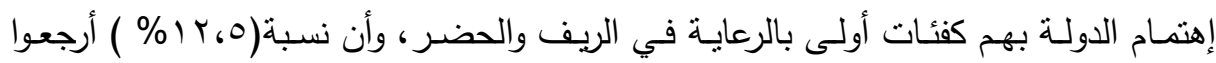

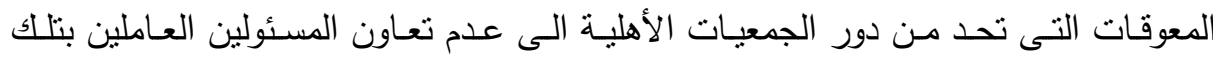
الجمعيات في الريف والحضر بيتضح من نتائج الدراسة الميدانية أن جميع المطلقات والأرامل لئل

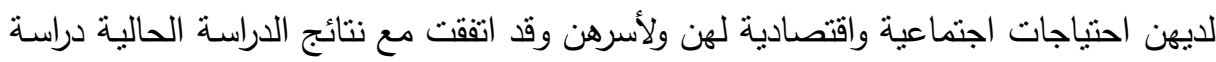

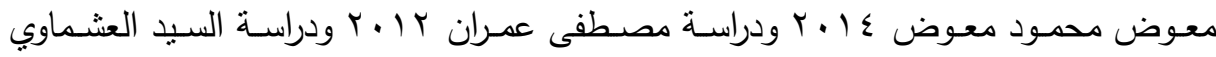


با ـ ب ودراسـات اخرى كثيـرة وتوصـل البـاحثون لمجموعـة مـن التوصـيات كـان مـن أهمها

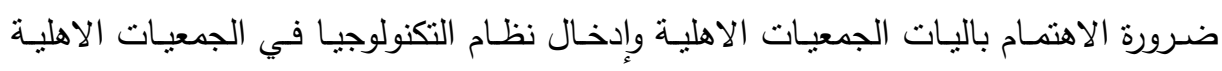

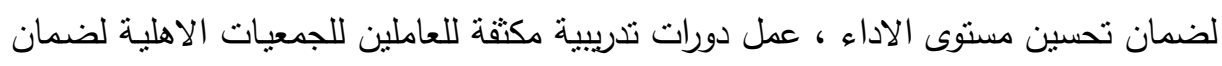
تحسين مستوى الاداء.

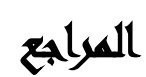

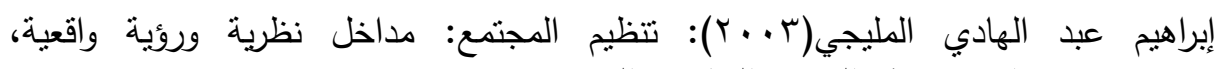
الإسكندرية، المكتب الجامعي الحديث.

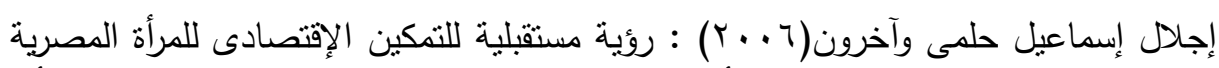

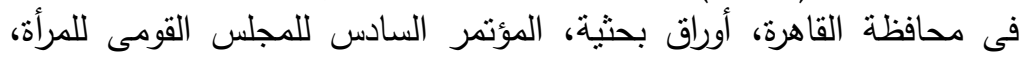
القاهرة.

أحمد بدر (1 (1) ب): البحث العلمي ومناهجه، الطبعة التاسعة، المكتبة الأكاديمية، القاهرة. أحمد زكى بدوى( (19 ( )): معجم مصطلحات العلوم الاجتماعية، مكتبة لبنان، بيروت.

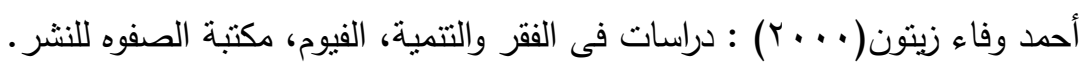

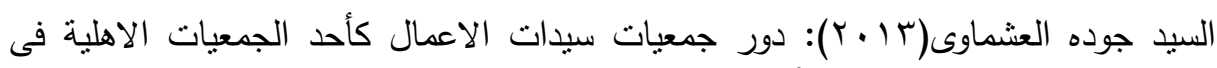

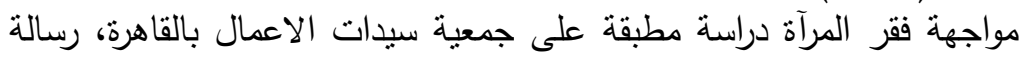

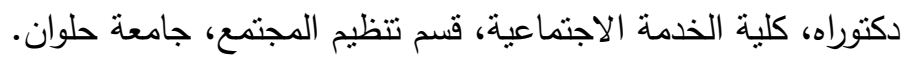

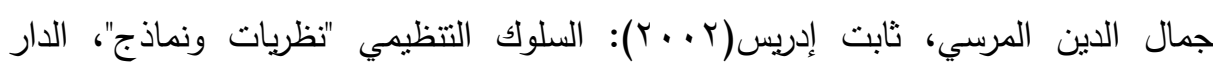
الجامعية، الإسكندرية.

سامية الخشاب(ب99 (19): النظرية الاجتماعية ودراسة الاسرة، القاهرة، دار المعارف.

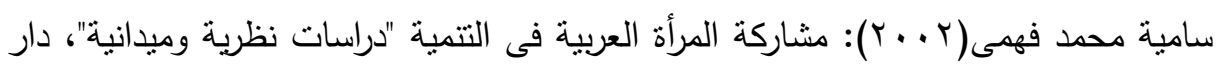
المعرفة، الاسكندرية.

صادق عادل(1 (99 )): امرأة في محنة، كتاب اليوم الطبي ، مؤسسة أخبار اليوم، القاهرة.

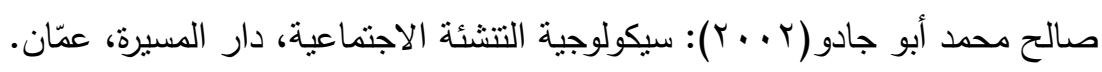
طلعت مصطفى السروجى(9 . . ץ) : رأس المال الاجتماعى، القاهرة، مكتبة الانجلو. 
عبد الحليم رضا عبد العال وآخرون(997 (): تتظيم المجتمع "أجهزة ومجالات وحالات"، مكتبة نهضة الثرق، القاهرة.

عبد الحليم رضا عبد العال وآخرون(997 (1)): تتظيم المجتمع أجهزة ومجالات، القاهرة، مكتبة النهضة.

عبد المطلب القريطي(99199): في الصحة النفسية، القاهرة، دار الفكر العربي. عبير عبد المنعم(9.. †): علم الاجتماع وتتمية الوعي الاجتماعي بالمتغيرات العالمية والمحلية، المكتبة العصرية للنشر والتوزيع، القاهرة.

على الطهطاوى( . . ب ؟): الخلع فى ضوء الكتاب والسنه، القاهرة، المكتبة المحمودية.

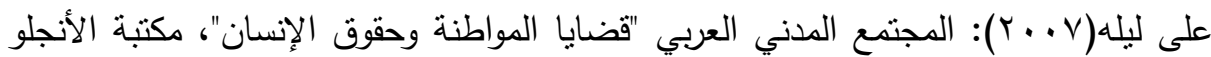

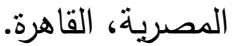

فرخندة حسن( . . ب): نهضة مصر "المرأة والمواطنة والتتمية"، المؤتمر القومي الأول للمرأة، المجلس القومي للمرأة، القاهرة.

محمد الجوهري(991 (): علم الاجتماع التطبيقي، دار المعرفة الجامعية، الإسكندرية. محمد عاطف غيث(990 (190): قاموس علم الاجتماع، الاسكندرية، دار المعرفة الاجتماعية. محمد عويس( ( . ץ): البحث العلمى فى الخدمة الاجتماعية (الدراسة والتتخص فى بحوث

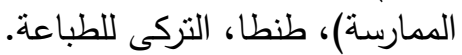

Robert L. Barker (1987): The Social Work Dictionary, Wash-ington, National Association of Social Workers Press.

Robert L. Barker (1999): The Social Work Dictionary 3rd ed., Slier Spring, The N.A.S.W Press. 
إجلال إسماعيل حلمي وآخرون

\title{
THE ROLE OF CIVIL SOCIETIES IN SATISFYING SOCIAL AND ECONOMIC NEEDS OF THE DIVORCED AND WIDOWED WOMEN
}

Helmy, Iglal, I. ${ }^{(1)}$; Amer, M. E. A. ${ }^{(2)}$ and Ahmed, Abeer, A. S. ${ }^{(3)}$ 1) Faculty of Arts, Ain Shams University 2) Higher Institute for Social Work- Kafr El - Sheikh 3) Higher Institute for Social Work- Bnha

\begin{abstract}
One of the most important fields that civil societies are concerning with is the field of developing woman in general, and the divorced or widowed woman in particular, particularly, those are working in multiple fields such as the empowerment field. This means that woman should be provided with training, habilitation, and work opportunities. The second field is the rights defensive field that assesses the lawful literacy of rights, duties, and defense for marginalized woman. The third field is the traditional field which is responsible for introducing services of social care and welfare.

This research aims to identify the role of civil societies in satisfying the social and economic needs of widows. The study was applied on a sample of (40) widows and divorced women in al Qalubia governorate who benefit from social insurance in both rural and urban areas. The study came to the following conclusions:

- Widows and divorced women in rural and urban areas, have social and economic needs.

- Civil societies play a role in satisfying the social and economic needs of widows and divorced women

- The majority of the participants are not satisfied with the services pervaded by the civil societies.

- There are obstacles that limit the role of civil societies in helping widows and divorced women.
\end{abstract}

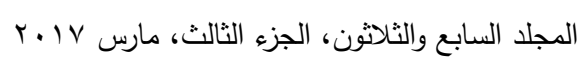


- Widows in rural as well as urban areas are in need to social services represented in educational, health, social relations needs; while the economic needs are embodied in having a fixed stable income and the materialistic and concrete aids, in addition to small enterprises.

Researchers have set a group of suggestions and recommendations that can improve the role of civil societies to satisfy the benefitted categories, particularly, the widows and divorced women. The study suggests including the technology system in civil societies, improving as well the mechanisms of these associations through holding training courses for employees, improving presented service and activities through making scientific researches to identify the actual needs of the benefitted categories. 